\title{
Added Value of Clinical Sequencing: WGS-Based Profiling of Pharmacogenes
}

\author{
Sylvan M. Caspar ${ }^{1,2}$, Timo Schneider ${ }^{1}$, Janine Meienberg ${ }^{1}$ and Gabor Matyas ${ }^{1,3, *(1)}$ \\ 1 Center for Cardiovascular Genetics and Gene Diagnostics, Foundation for People with Rare Diseases, \\ 8952 Schlieren-Zurich, Switzerland; caspar@genetikzentrum.ch (S.M.C.); \\ praktikanten@genetikzentrum.ch (T.S.); meienberg@genetikzentrum.ch (J.M.) \\ 2 Laboratory of Translational Nutrition Biology, Department of Health Sciences and Technology, ETH Zurich, \\ 8603 Schwerzenbach, Switzerland \\ 3 Zurich Center for Integrative Human Physiology, University of Zurich, 8057 Zurich, Switzerland \\ * Correspondence: matyas@genetikzentrum.ch; Tel.: +41-43-433-86-86
}

Received: 7 February 2020; Accepted: 24 March 2020; Published: 26 March 2020

check for updates

\begin{abstract}
Although several pharmacogenetic (PGx) predispositions affecting drug efficacy and safety are well established, drug selection and dosing as well as clinical trials are often performed in a non-pharmacogenetically-stratified manner, ultimately burdening healthcare systems. Pre-emptive PGx testing offers a solution which is often performed using microarrays or targeted gene panels, testing for common/known PGx variants. However, as an added value, whole-genome sequencing (WGS) could detect not only disease-causing but also pharmacogenetically-relevant variants in a single assay. Here, we present our WGS-based pipeline that extends the genetic testing of Mendelian diseases with PGx profiling, enabling the detection of rare/novel PGx variants as well. From our in-house WGS (PCR-free 60× PE150) data of 547 individuals we extracted PGx variants with drug-dosing recommendations of the Dutch Pharmacogenetics Working Group (DPWG). Furthermore, we explored the landscape of DPWG pharmacogenes in gnomAD and our in-house cohort as well as compared bioinformatic tools for WGS-based structural variant detection in CYP2D6. We show that although common/known PGx variants comprise the vast majority of detected DPWG pharmacogene alleles, for better precision medicine, PGx testing should move towards WGS-based approaches. Indeed, WGS-based PGx profiling is not only feasible and future-oriented but also the most comprehensive all-in-one approach without generating significant additional costs.
\end{abstract}

Keywords: CYP2D6; DPWG; gnomAD; next-generation sequencing; precision medicine; pharmacogenetics; PGx; whole-genome sequencing

\section{Introduction}

Pharmacogenetics is primarily concerned with how genetic variation affects individual drug response [1]. In the current genomics era, technological advances allow the unprecedented implementation of pharmacogenetics and its importance is becoming increasingly evident. Less than $10 \%$ of drugs reach approval [2]; the costs of drug development, following Eroom's law, have risen to almost $€ 3$ bn per marketed drug [3]; healthcare costs are increasing; and the number of annual deaths and costs due to adverse drug events (ADEs) are estimated to be 197'000 and €79 bn, respectively, in the EU [4]. In the US, the annual cost caused by nonoptimized medical therapy is estimated to be approximately $\$ 530$ bn [5]. In light of these facts, there is a need for improvement in both drug development and healthcare systems. Pharmacogenetics may provide a solution to tackle these issues in the form of pre-emptive pharmacogenetic (PGx) testing for drug selection and dosing as well as PGx-based stratification of clinical trials. While in oncology pharmacogenetics has already 
been implemented to identify patients with a higher chance to benefit from a treatment according to tumor-driver variants [6], in non-cancer cases drug selection and dosing as well as clinical trials are often performed in a non-pharmacogenetically-stratified manner.

For pre-emptive PGx testing, a variety of commercially available providers and assays exist, including Abomics (abomics.fi), bio.logis (biologis.de), PharmacoScan (thermofischer.com), Sonogen (sonogen.eu), and VeriDose (agenabio.com) which use PCR- and/or microarray-based techniques. In addition, targeted gene panels using next-generation sequencing (NGS) are available, such as the AmpliSeq Pharmacogenomics Research Panel (thermofisher.com), PGRNseq [7], and the VeraCode ADME Core Panel (illumina.com). The focus mostly lies on screening for the most common and well described (known) PGx variants affecting pharmacokinetics or -dynamics, often referred to as star $\left(^{*}\right)$ alleles. For translation of the relevant genotypes to actionable PGx recommendations, guidelines of international experts are frequently invoked, such as the Dutch Pharmacogenetics Working Group (DPWG) [8] or the Clinical Pharmacogenetics Implementation Consortium (CPIC) [1]. As pre-emptive PGx testing is not yet routine in most clinics, several trials are currently evaluating cost-effectiveness and patient outcomes, including (i) the EU-funded PREPARE clinical study [9], in which microarray-based testing is used, enabling easy-to-use and platform-independent decision support by providing results on the Medication Safety Code system [10]; (ii) the CLIPMERGE PGx Program [11]; (iii) the eMERGE-PGx project [12], the latter two deploying PGRNseq [7], enabling the screening of selected pharmacogenes and integrating the results of PGx profiling into electronic health records (EHRs).

NGS-based approaches have the advantage of additionally detecting novel (yet-unknown) and rare but pharmacogenetically-relevant variants, which may contribute to interindividual variability, allowing better PGx profiling and personalized medicine [13]. Indeed, the increasingly used whole-exome sequencing (WES) and especially whole-genome sequencing (WGS) may not only be used for the diagnosis of Mendelian diseases, but also represent an untapped data source for pre-emptive PGx testing due to sequencing all (known) genes to a large extent, including pharmacogenes [14]. However, WGS-based PGx profiling is not yet common and the true contribution of common/known PGx variants compared to rare/novel (and thus usually not tested) variants is unknown. In addition, due to short-read-related alignment ambiguities in repetitive or homologous genomic regions, accurate variant calling in the cytochrome P450 (CYP) enzyme superfamily, members of which are responsible for the biotransformation of $\sim 70 \%-80 \%$ of all drugs, is challenging. The most notable example is the pharmacogene CYP2D6, which is highly polymorphic and homologous to its two pseudogenes and metabolizes $\sim 25 \%$ of all drugs in clinical use [15]. As in such homologous/repetitive genomic regions, standard short-read-based alignment and variant calling pipelines may not reliably detect all pharmacogenetically-relevant variants, specialized software tools have been introduced to call difficult-to-detect variants, including CYP2D6 structural variants (SVs) [15-18]. These specialized short-read-based software tools, however, have not been independently evaluated.

To address these issues, we present our first-of-its-kind pipeline from raw short-read WGS data to diagnosis and PGx profiling, thereby implementing drug-dosing recommendations according to the DPWG guidelines. Furthermore, we assess relative allele frequencies of both common/known PGx and rare/novel loss-of-function (LOF) variants in current DPWG pharmacogenes in the largest publicly available population-based reference dataset gnomAD (v2.1.1 and v3) and in our large in-house WGS cohort as well as evaluate the accuracy of WGS-based CYP2D6 (structural) variant detection and discuss the future perspectives of PGx implementation.

\section{Results}

\subsection{Pipeline from Raw Data to Molecular Genetic Diagnosis, PGx Report, and Medication Safety Card (MSC)}

Our WGS-based clinical sequencing pipeline has been expanded to include PGx profiling (Figure 1a). For this, pharmacogenetically-relevant variants were extracted from WGS data and 
interpreted according to the quarterly-updated DPWG guidelines. Similar to the PREPARE trial $[9,10]$, our expanded pipeline outputs individualized treatment recommendations on a PGx report (Figure 1b) as well as on a PGx profile (Figure 1c) in credit card format (medication safety card, MSC), both of which can accordingly be used for better patient care. Currently, our pipeline analyzes the most established 45 single nucleotide variants (SNVs) and insertions/deletions (indels) in 11 genes as well as an $H L A-B * 5701$ tagging variant and the deletion/duplication of CYP2D6, providing DPWG recommendations for $77+X(X=$ oral contraceptives) drugs (hereafter referred to as DPWG variants). As additional pharmacogenetically-relevant variants are expected to be described in the future, the MSC contains a QR code, providing access to an appropriate portal (accessible online or via app), containing up-to-date recommendations for personalized drug selection and dosing. Thus, our expanded pipeline not only allows WGS-based diagnosis but also individualized PGx recommendations for drug selection and dosing.

a

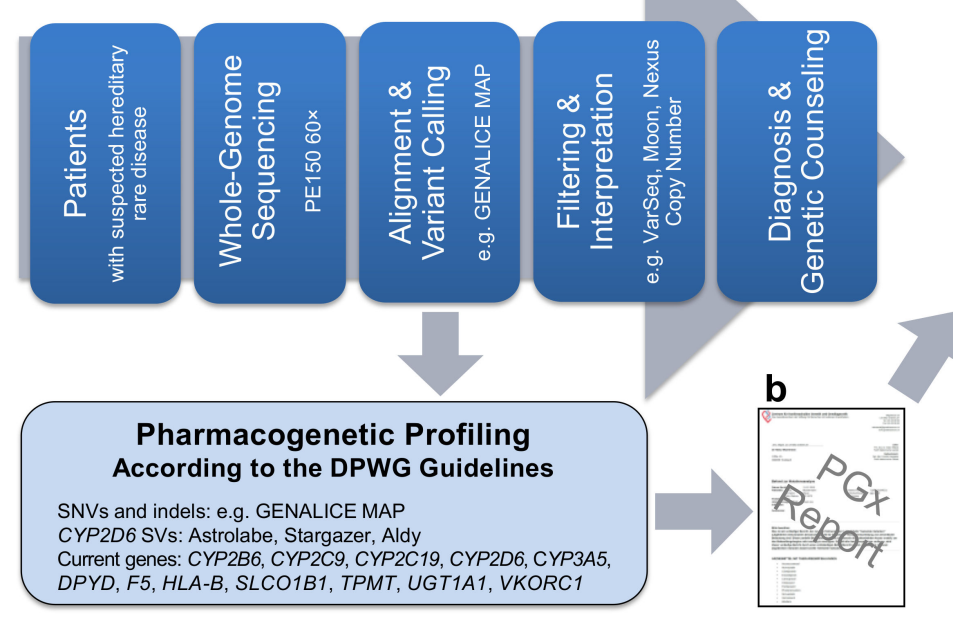

c

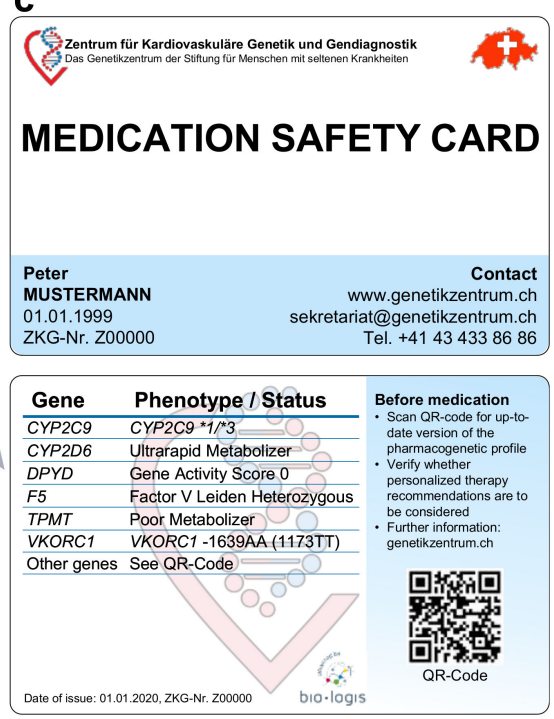

Figure 1. Our whole-genome sequencing (WGS)-based pharmacogenetic (PGx) profiling. (a) Pipeline from whole-genome sequencing data to (b) PGx report and (c) individualized PGx profile in credit card format (Medication Safety Card). Abbreviations: DPWG, Dutch Pharmacogenetic Working Group; indels, insertions/deletions; SNVs, single nucleotide variants; SVs, structural variants.

\subsection{Evaluation of $P G x$ Variants in gnom $A D$ and Our In-House Cohort}

We explored the landscape of 11 DPWG pharmacogenes as well as an HLA-B*5701 tagging variant, in gnomAD exomes v2.1.1, gnomAD genomes v3, and our in-house WGS cohort. For this, we calculated the relative allele frequencies not only of DPWG but also of LOF/missense variants that most likely affect the function of pharmacogenes as well. In total, we detected 966, 637, and 51 pharmacogenetically most likely relevant variants in gnomAD exomes v2.1.1, gnomAD genomes v3, and our in-house WGS cohort, respectively (Table 1, Supplementary Table S1). The 45 DPWG variants account for the vast majority ( $98 \%$, ranging from $59 \%$ to $100 \%$ ) of detected alleles, depending on gene, dataset, and subpopulation (Supplementary Table S1). Notably, 55\%, 51\%, and $49 \%$ of the DPWG variants occurred with a minor allele frequency (MAF) between $0.1 \%$ and $5 \%$, while $25 \%, 31 \%$, and $38 \%$ of the DPWG variants occurred with a MAF above 5\% in gnomAD exomes v2.1.1, gnomAD genomes v3, and our in-house cohort, respectively (Table 1, Figure 2). In contrast, 99.8\%, 98.4\%, and 100.0\% of the novel (i.e., not listed in ClinVar, HGMD or PharmGKB) LOF/missense variants can be classified as rare, occurring with a MAF $<0.1 \%$. The full table of retained variants is listed in Supplementary Table S1. Furthermore, in our in-house WGS cohort of 547 genomes we detected 37 of the 45 DPWG variants (Table 1), on average, 6 of which occurred per genome (Supplementary Figure S1). 
Table 1. Number (n) and proportions (\%) of DPWG pharmacogene variants detected in gnomAD and our in-house WGS cohort.

\begin{tabular}{|c|c|c|c|c|c|c|}
\hline Cohort & \multicolumn{2}{|c|}{$\begin{array}{c}\text { gnomAD Exomes } \\
\text { v2.1.1 }\end{array}$} & \multicolumn{2}{|c|}{$\begin{array}{l}\text { gnomAD Genomes } \\
\text { v3 }\end{array}$} & \multicolumn{2}{|c|}{$\begin{array}{l}\text { In-House WGS } \\
\text { Cohort }\end{array}$} \\
\hline Cohort Size & \multicolumn{2}{|c|}{ 125'748 Exomes } & \multicolumn{2}{|c|}{ 71'702 Genomes } & \multicolumn{2}{|c|}{547 Genomes } \\
\hline $\begin{array}{c}\text { Novel LOF/missense variants } \\
\text { (not in ClinVar/HGMD/PharmGKB) }{ }^{1}\end{array}$ & $\mathbf{n}$ & $\%$ & $\mathbf{n}$ & $\%$ & $\mathbf{n}$ & $\%$ \\
\hline Total variants (alleles) & $823\left(4^{\prime} 214\right)$ & 100.0 & $512\left(6^{\prime} 940\right)$ & 100.0 & $10(10)$ & 100.0 \\
\hline $\mathrm{MAF}>5 \%$ & 0 & 0.0 & 0 & 0.0 & 0 & 0.0 \\
\hline $0.1 \%<\mathrm{MAF}<5 \%$ & 2 & 0.2 & 8 & 1.6 & 0 & 0.0 \\
\hline $\mathrm{MAF}<0.1 \%$ & 821 & 99.8 & 504 & 98.4 & 10 & 100.0 \\
\hline $\begin{array}{l}\text { Known LOF/missense variants } \\
\text { (in ClinVar/HGMD/PharmGKB) }{ }^{1}\end{array}$ & n & $\%$ & n & $\%$ & $\mathbf{n}$ & $\%$ \\
\hline Total variants (alleles) & $103\left(5^{\prime} 584\right)$ & 100.0 & $80\left(2^{\prime} 706\right)$ & 100.0 & $4(17)$ & 100.0 \\
\hline $\mathrm{MAF}>5 \%$ & 0 & 0.0 & 0 & 0.0 & 0 & 0.0 \\
\hline $0.1 \%<\mathrm{MAF}<5 \%$ & 5 & 4.9 & 3 & 3.7 & 1 & 25.0 \\
\hline $\mathrm{MAF}<0.1 \%$ & 98 & 95.1 & 77 & 96.3 & 3 & 75.0 \\
\hline DPWG variants & $\mathrm{n}$ & $\%$ & $\mathbf{n}$ & $\%$ & $\mathbf{n}$ & $\%$ \\
\hline Total variants (alleles) & $40\left(375^{\prime} 331\right)$ & 100.0 & $45\left(487^{\prime} 758\right)$ & 100.0 & $37\left(4^{\prime} 162\right)$ & 100.0 \\
\hline $\mathrm{MAF}>5 \%$ & 10 & 25.0 & 14 & 31.1 & 14 & 37.8 \\
\hline $0.1 \%<\mathrm{MAF}<5 \%$ & 22 & 55.0 & 23 & 51.1 & 18 & 48.7 \\
\hline $\mathrm{MAF}<0.1 \%$ & 8 & 20.0 & 8 & 17.8 & 5 & 13.5 \\
\hline
\end{tabular}

${ }^{1} \mathrm{LOF} /$ missense variants in $H L A-B$ were not considered due to short-read-related alignment ambiguities. Abbreviations: DPWG, Dutch Pharmacogenetics Working Group; LOF, loss of function; MAF, minor allele frequency.

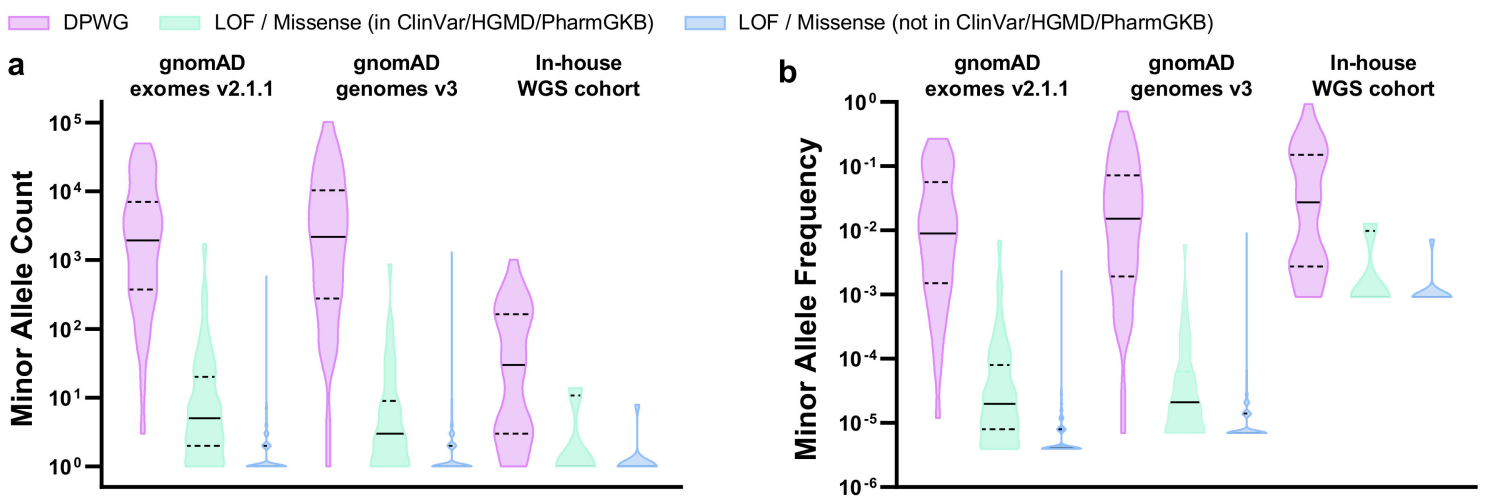

Figure 2. Violin plots showing the distributions of (a) minor allele counts and (b) minor allele frequencies in 11 DPWG genes (CYP2B6, CYP2C9, CYP2C19, CYP2D6, CYP3A5, DPYD, F5, SLCO1B1, TPMT, UGT1A1, VKORC1) and an HLA-B*5701 tagging variant in gnomAD exomes v2.1.1, gnomAD genomes v3, and our in-house WGS cohort. Horizontal lines indicate median, horizontal dashed lines indicate quartiles. Plots were generated using Graphpad Prism 8 (Graphpad Software, CA, USA). Abbreviations: DPWG, Dutch Pharmacogenetics Working Group; LOF, loss of function.

For LOF/missense and DPWG variants, including the respective DPWG star $\left(^{*}\right)$ alleles, we calculated relative allele frequencies and compared them among the three databases (Figure 3). To infer the number of wildtpye $\left({ }^{*} 1\right)$ alleles, we made the assumption that variants detected in one gene occur in trans. In general, the inferred wildtype allele is the most prominent allele, however, in the genes CYP2D6 (in-house genomes), CYP2B6 (gnomAD and in-house genomes), and CYP3A5 (gnomAD and in-house genomes), known DPWG variants are more frequent than the inferred wildtype (Figure 3a,d,e). Specifically, the DPWG variants CYP2D 6*3, ${ }^{*} 4,{ }^{*} 6,{ }^{*} 8,{ }^{*} 9,{ }^{*} 10,{ }^{*} 14,{ }^{*} 17,{ }^{*} 41$ and CYP $2 B 6^{*} 4, * 5,4, * 18$, when combined, amount to more alleles than the respective inferred wildtype alleles CYP2B6*1 and CYP2D6*1. In addition, the CYP3A5*3 allele (NM_000777.4: c.219-237A>G, intronic splicing variant; www.pharmgkb.org/vip/PA166170041) is the most common allele of CYP3A5, constituting the majority of alleles in gnomAD genomes and our in-house WGS cohort, whereas it is not 
captured in WES-based gnomAD exomes. Similarly, the variants CYP2C19*17 (c.-806C>T, promoter), VKORC1*2 (c.174-136C>T, deep intronic), and UGT1A1*28*37 (c.-41_-40dup/ c.-43_-40dup) are not present in gnomAD exomes (cf. CYP2C19*17 and $C Y P 3 A 5^{*} 3$ are not covered in the assessed in-house WES samples as well). Note that $60 \times$ WGS performed better (i.e., resulted in better coverage) than $30 \times$ WGS and 100× WES in all investigated categories (Supplementary Figure S2).

CYP2D6*10 CYP2D6*4 CYP2D6*41

- CYP2D6*17 CYP2D6*9 CYP2D6*3

- CYP2D6*6 LOF $^{\dagger}=$ CYP2D6*14 $^{\dagger}$

- LOF $^{\S}$ CYP2D6*8

WT $61.9 \% \quad$ WT $51.2 \% \quad$ WT $44.9 \%$

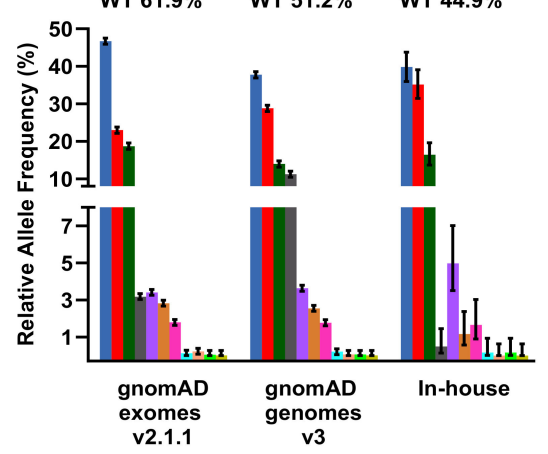

C

$\begin{array}{ll}\text { CYP2C9*2 }^{*} & \begin{array}{l}\text { CYP2C9*3 } \\ \mathrm{LOF}^{\S}\end{array} \text { CYP2C9*11 }^{\text {CYP2C9*5 }} \text { LOF }^{\dagger}\end{array}$
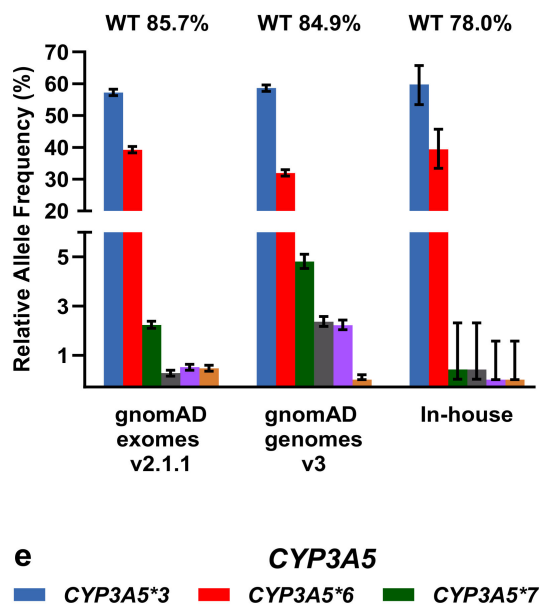

- LOF $^{\dagger}$ LOF $^{\S}$

WT $97.6 \% \quad$ WT $20.1 \% \quad$ WT $3.9 \%$

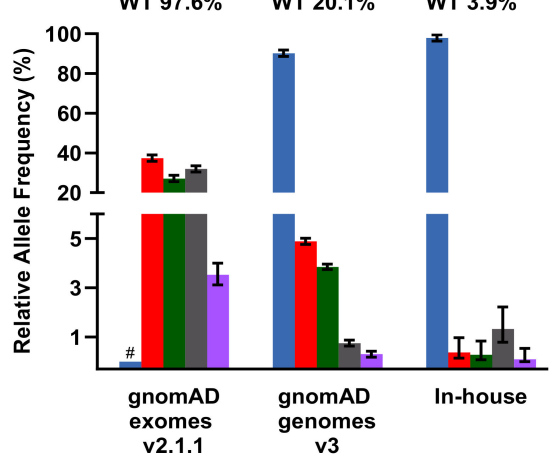

b $\quad$ CYP2C19

- CYP2C19*17 CYP2C19*2 - CYP2C19*9

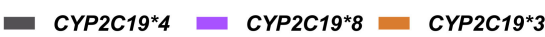

- CYP2C19*10 $=$ CYP2C19*6 $^{*}$ LOF $^{\S}$

- CYP2C19*5 LOF $^{\dagger}$

WT $88.9 \% \quad$ WT $62.2 \% \quad$ WT $63.7 \%$

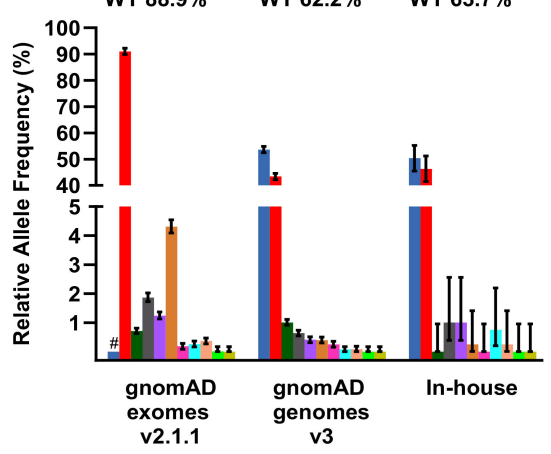

d $\quad$ CYP2B6

- CYP2B6*9 CYP2B6*4 CYP2B6*5.1

CYP2B6*18 LOF $^{\S}=$ CYP2B $^{*} 5.2$

- LOF $^{\dagger}$

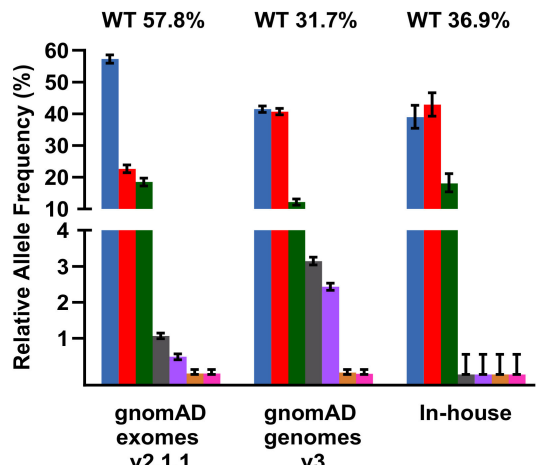

f DPYD

- $D P Y D(\mathrm{rs} 56038477)=\mathrm{LOF}^{\dagger}=D P Y D^{\star} 2 A$

- DPYD (rs67376798) LOF ${ }^{\S}$ DPYD*13

WT $96.9 \% \quad$ WT $96.7 \% \quad$ WT $96.8 \%$

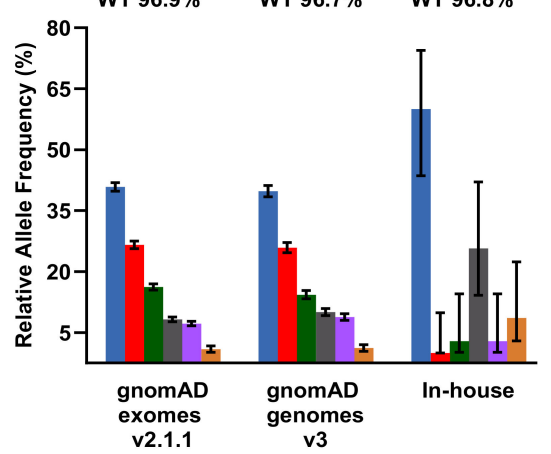

Figure 3. Cont. 
g
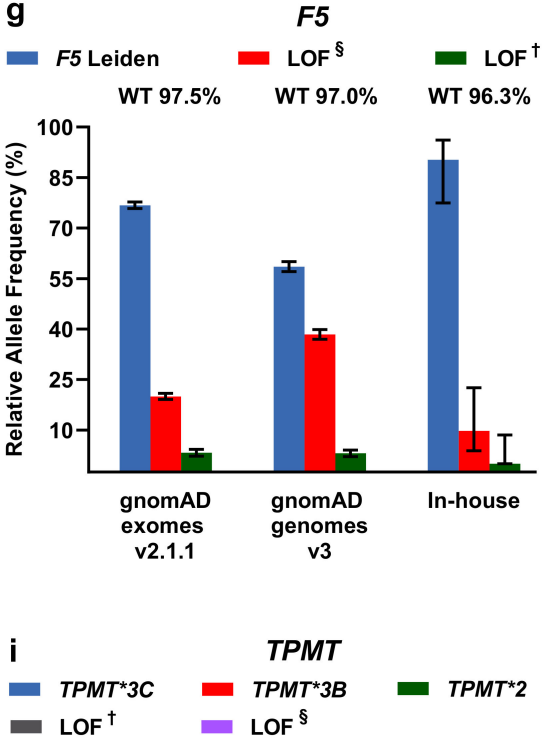

WT $94.1 \%$ WT $92.7 \%$ WT $93.0 \%$

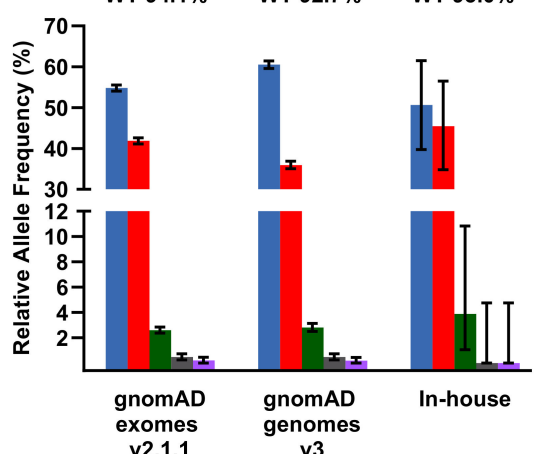

v3

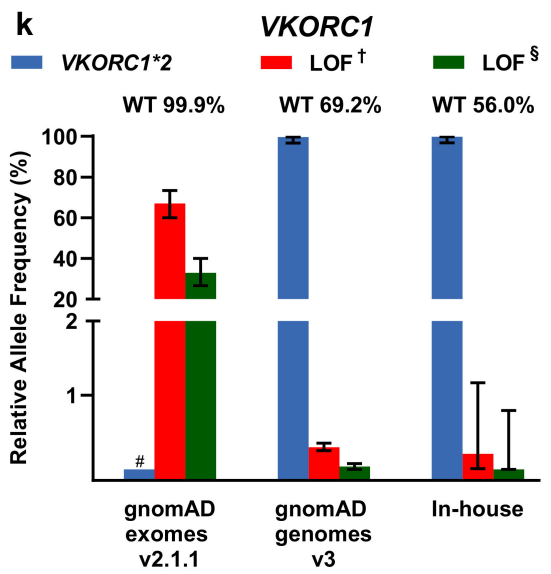

h

\begin{tabular}{ll} 
SLCO1B1*5 & \multicolumn{2}{c}{ CO1B1 } \\
LOF $^{\S}$ & LOF $^{\dagger}$
\end{tabular}

WT $87.8 \%$ WT $87.0 \%$ WT $87.4 \%$

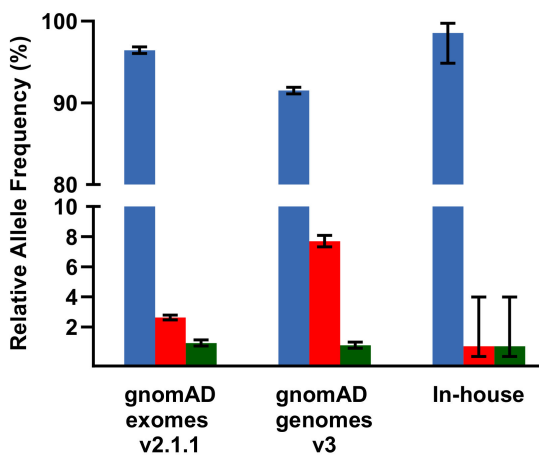

j UGT1A1

UGT1A1*28 UGT1A1*37 UGT1A1*6

UGT1A1*27 LOF $^{\S}=$ LOF $^{\dagger}$

WT $97.9 \% \quad$ WT $62.9 \% \quad$ WT $63.6 \%$

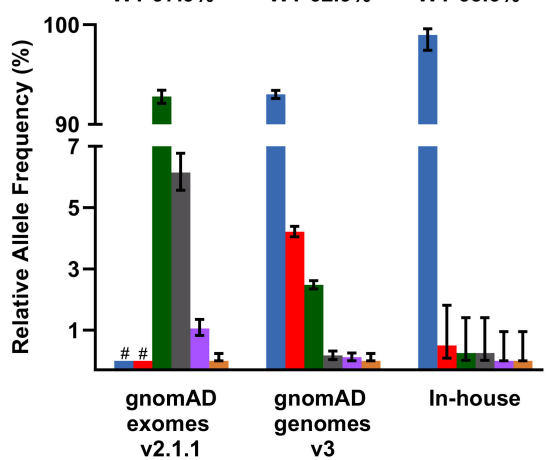

Figure 3. (a-k) Relative allele frequencies of pharmacogenetically-relevant variants in gnomAD exomes and genomes as well as in our in-house WGS cohort. The percentage of corresponding wildtype (WT) alleles is denoted. Note that wildtype status was inferred under the assumption that the listed variants detected in the same gene occur in trans and that no additional pharmacogenetically- relevant variant occurs at the wildtype allele. Note that some of the DPWG variants are not covered in gnomAD exomes (denoted with \# in the graphs of CYP2C19*17, CYP3A5*3,UGT1A1*28,*37, and VKORC1*2). Error bars indicate $95 \%$ confidence intervals. ${ }^{\dagger}$ Loss-of-function (LOF) variants listed in HGMD, ClinVar, or PharmGKB. ${ }^{\S}$ LOF variants not listed in HGMD, ClinVar, or PharmGKB in the context of drug response. 
Other less common DPWG variants, however, are not present in our primarily Caucasian in-house WGS cohort, likely due to the relatively small cohort size or because variants are predominantly detected in different subpopulations such as the CYP2C9*5 allele in individuals of African descent (Supplementary Table S1). In contrast, the variants $C Y P 2 C 9^{*} 2$ and $C Y P 2 C 9^{*} 3$ are significantly more frequent in the European subpopulations of gnomAD exomes v2.1.1 and gnomAD genomes v3 compared to the African subpopulation (Supplementary Table S1). Overall, however, the contributions are comparable among the three databases and with previous estimates $[13,19]$.

Moreover, in our in-house cohort of 547 genomes, we screened for DPWG and LOF/missense variants occurring in the same gene. In our cohort, 19 individuals harbor such co-occurring variants, in all but one of which the additional LOF/missense variant may also cause decreased or no enzyme function. One individual harbors not only the allele $C Y P 2 C 19 * 17$ (c.-806C $>$ T) causing the ultrarapid metabolizer phenotype, but also the allele $\mathrm{CYP} 2 \mathrm{C} 19^{*} 2$ causing poor metabolizer phenotype as well as the hitherto undescribed $C Y P 2 C 19$ splicing variant c.643-2A $>$ T likely disrupting gene function (cf. possibly LOF). Although phasing was not possible for these variants, not considering the novel splicing variant could result in a misclassification of the CYP2C19 metabolizer status. Furthermore, because many of our patients might be treated with an anticoagulant due to their cardiovascular phenotype, we screened for CYP2C9 and VKORC1 variants affecting phenprocoumon/warfarin dosing. Thereby, we detected 113 individuals homozygous for the high phenprocoumon/warfarin sensitivity variant $V K O R C 1^{*} 2$ as well as 11 and 2 individuals homozygous for the poor metabolizer variants CYP2C9*2 and CYP2C9*3, respectively. We also detected 5 individuals homozygous for VKORC1*2 and $C Y P 2 C 9^{*} 2$, requiring a reduction of the phenprocoumon/warfarin starting dose to $\sim 35 \%$ compared to $V K O R C 1 * 1 / C Y P 2 C 9^{*} 1$ individuals [20].

\subsection{Comparison of CYP2D6 Calling Tools from WGS Data}

Three command-line-based bioinformatic tools, Astrolabe (previously Constellation) [15], Aldy [16], and Stargazer [17] were used to call CYP2D6 variants, including SVs. Using downloaded genetic reference data, we compared the CYP2D6 variant calls of these three tools to the GeT-RM 2019 consensus genotypes [21]. As shown in Table 2, of 21 samples Stargazer called 11, Astrolabe 12, and Aldy 19 correctly (note that for NA18524 Aldy detected all existing star allels but not in the right diplotype). The number of incorrect calls seems to be linked to samples with more than two star alleles. In the sample NA18519, Aldy and Stargazer detected the ${ }^{*} 106$ star allele listed as ${ }^{*} 1$ in the GeT-RM 2019 consensus genotype, which we confirmed as *106 by manual evalaution of the BAM file using the Integrative Genomics Viewer [22]. As Aldy reached the highest accuracy, we analyzed 547 WGS samples (primarily European descent) using this tool (Supplementary Figure S3). While the two most frequently detected CYP2D 6 star alleles $\left({ }^{*} 1,351\right.$ alleles; ${ }^{*} 2,187$ alleles) encode a normally functioning enzyme, the most frequently detected alleles with decreased ( ${ }^{*} 41,93$ alleles; ${ }^{*} 10,25$ alleles) or no function $\left({ }^{*} 4,123\right.$ alleles; ${ }^{*} 68+4,71$ alleles; $* 5,30$ alleles) together amount to approximately $30 \%$ of the CYP2D6 star alleles in our cohort.

The three calling tools are comparable regarding hardware requirements, runtime, and disk footprint of output files, albeit Stargazer requires a GATK-DepthOfCoverage format file (Supplementary Figure S4). Aldy and Stargazer accept WGS, WES, and targeted sequencing data as input (cf. WGS offering best accuracy), while Astrolabe requires WGS data (i.e., unsuitable for WES). 
Table 2. Comparison of CYP2D6 callers output, in form of star alleles (single nucleotide variants, small insertions/deletions, structural variations), from 21 publicly available short-read WGS samples.

\begin{tabular}{|c|c|c|c|c|}
\hline $\begin{array}{l}\text { Reference } \\
\text { Samples }\end{array}$ & $\begin{array}{l}\text { GeT-RM } \\
\text { Consensus } \\
\text { Genotype } 2019\end{array}$ & $\begin{array}{l}\text { Astrolabe } \\
\text { v0.8.6.1 }\end{array}$ & $\begin{array}{l}\text { Aldy } \\
\text { v2.2.3 }\end{array}$ & $\begin{array}{l}\text { Stargazer }{ }^{1} \\
\text { v1.0.7 }\end{array}$ \\
\hline HG00436 & ${ }^{*} 2 \times 2 /{ }^{*} 71$ & $* 2 / * 71$ & $* 2 \times 2 /^{*} 71$ & ${ }^{*} 1 /{ }^{*} 83+{ }^{*} 2$ \\
\hline NA07029 & ${ }^{*} 1 / * 35$ & $* 1 / * 35$ & ${ }^{*} 1 / * 35$ & ${ }^{*} 1 / * 35$ \\
\hline NA18959 & ${ }^{*} 2 / * 36+{ }^{*} 10$ & $* 2 /{ }^{*} 10$ & $* 2 / * 36+{ }^{*} 10$ & ${ }^{*} 2 /{ }^{*} 36+{ }^{*} 10$ \\
\hline NA19109 & $* 2 \times 2 / * 29$ & $* 2 / * 29$ & $* 2 \times 2 / * 29$ & ${ }^{*} 29 / * 83+* 2$ \\
\hline NA21781 & ${ }^{*} 2 \times 2 /{ }^{*} 68+{ }^{*} 4$ & $* 2 / 4^{2}$ & ${ }^{*} 2 \times 2 /{ }^{*} 68+{ }^{*} 4$ & ${ }^{*} 4 N+{ }^{*} 4 /{ }^{*} 68+{ }^{*} 4$ \\
\hline NA12878 in-house & ${ }^{*} 3 /\left({ }^{*} 68\right)+{ }^{*} 4$ & $* 3 /{ }^{*} 4^{3}$ & ${ }^{*} 3 /{ }^{*} 68+* 4$ & $* 3 /{ }^{*} 4$ \\
\hline NA12873 & ${ }^{*} 1 / * 5$ & ${ }^{*} 1 / * 5$ & $* 5 /{ }^{*} 61^{4}$ & ${ }^{*} 1 /{ }^{* 5}$ \\
\hline NA18861 & $* 5 / * 29$ & $* 5 / * 29$ & $* 5 / * 29$ & ${ }^{*} 13 C{ }^{*} 29$ \\
\hline HG00589 & $* 1 / * 21$ & $* 1 / * 2$ & $* 1 / * 21$ & ${ }^{*} 1 /{ }^{*} 2$ \\
\hline NA19917 & ${ }^{*} 1 /{ }^{*} 40$ & ${ }^{*} 1 /{ }^{*} 40$ & ${ }^{*} 1 /{ }^{*} 40$ & ${ }^{*} 1 /{ }^{*} 40$ \\
\hline NA07019 & ${ }^{*} 1 /{ }^{*} 4$ & ${ }^{*} 1 /{ }^{*} 4$ & $* 1 /{ }^{*} 4$ & ${ }^{*} 1 /{ }^{*} 4$ \\
\hline NA12717 & ${ }^{*} 1 /{ }^{*} 1$ & ${ }^{*} 1 /{ }^{*} 1$ & ${ }^{*} 1 /{ }^{*} 1$ & ${ }^{*} 1 /^{*} 1$ \\
\hline HG00276 & ${ }^{*} 4 / * 5$ & ${ }^{*} 4 / * 4$ & ${ }^{*} 4 /{ }^{*} 5$ & ${ }^{*} 4 /{ }^{*} 5$ \\
\hline NA18524 & ${ }^{*} 1 /{ }^{*} 36 \times 2+{ }^{*} 10$ & ${ }^{*} 1 /{ }^{*} 10$ & ${ }^{*} 1+{ }^{*} 36 /{ }^{*} 36+{ }^{*} 10^{5}$ & ${ }^{*} 1 /{ }^{*} 10 \times 3$ \\
\hline NA18540 & $(* 36+)^{*} 10 / * 41$ & ${ }^{*} 41 /{ }^{*} 10^{3}$ & $* 36+10 / * 61+* 69$ & ${ }^{*} 10 \times 2 /{ }^{*} 41 \times 2$ \\
\hline NA12892 & $* 2 / * 3$ & $* 2 / * 3$ & ${ }^{*} 2 / * 3$ & $* 2 / * 3$ \\
\hline NA07348 & ${ }^{*} 1 /{ }^{*} 6$ & ${ }^{*} 1 /{ }^{*} 6$ & ${ }^{*} 1 /{ }^{*} 6$ & ${ }^{*} 1 /{ }^{*} 6$ \\
\hline NA18519 & ${ }^{*} 1 /{ }^{*} 29^{6}$ & $* 1 / * 29$ & ${ }^{*} 106 /{ }^{*} 29$ & ${ }^{*} 106 /{ }^{*} 29$ \\
\hline NA18966 & ${ }^{*} 1 /{ }^{*} 2$ & ${ }^{*} 1 /{ }^{*} 2$ & ${ }^{*} 1 / * 2$ & ${ }^{*} 1 /{ }^{*} 2$ \\
\hline NA18992 & $* 1 / * 5$ & $* 1 / * 5$ & $* 1 / * 5$ & ${ }^{*} 1 /{ }^{*} 13 C$ \\
\hline NA19226 & $* 2 / * 2 \times 2$ & $* 2 / 2^{2}$ & $* 2 / * 2 \times 2$ & $* 2 / * 83+* 2$ \\
\hline Total true & & 12 & 19 & 11 \\
\hline Total false & & 9 & 2 & 10 \\
\hline
\end{tabular}

${ }^{1}$ Stargazer outputs additional possible star alleles, but only the main star alleles are shown in this table. ${ }^{2}$ Possible gene duplication detected by Astrolabe. ${ }^{3}$ Possible gene hybrid ${ }^{*} 68$ detected by Astrolabe. ${ }^{4}$ Aldy could not phase any major solution, only potential star $\left(^{*}\right)$ alleles. ${ }^{5}$ Aldy detected the existing variants, although not in the same diplotype as in the GeT-RM consensus genotype 2019, and thus we counted the call as correct. ${ }^{6}$ The allele *106 is not listed in the GeT-RM consensus genotype, but detected by Stargazer and Aldy and manually confirmed using the Integrative Genomics Viewer. Green and red colors denote correct and incorrect calls, respectively. SNVs and indels are denoted by ${ }^{*} 2,{ }^{*} 3,{ }^{*} 4,{ }^{*} 6,{ }^{*} 10,{ }^{*} 21,{ }^{*} 29,{ }^{*} 35,{ }^{*} 36,{ }^{*} 40,{ }^{*} 41,{ }^{*} 69,{ }^{*} 71,{ }^{*} 83,{ }^{*} 106$, and SVs by ${ }^{*} 4 N, * 5,{ }^{*} 13,{ }^{*} 61,{ }^{*} 68$.

\section{Discussion}

In this work, we outline our first-of-its-kind approach describing seamless integration of PGx profiling into our WGS-based diagnostic pipeline for rare Mendelian disorders, without generating significant additional costs. By analyzing our in-house WGS cohort as well as the largest publicly available population-based dataset gnom $A D$, we show that variants with DPWG recommendations comprise the vast majority of detected pharmacogene alleles and that individuals harbor at least one pharmacogenetically-actionable variant. Moreover, our results show that even the highly polymorphic, pseudogene-burdened pharmacogene CYP2D6 may be accurately genotyped using short-read WGS, indicating that it is suitable for both diagnostics and PGx profiling in a single assay. Several discussion points and conclusions emerge from our results.

First, once a diagnosis is provided, the next step for adequate disease management ideally is a targeted medical therapy, tailored to the individual's PGx predispositions, if available. In an optimal scenario, information regarding PGx predisposition is available prior to medical therapy. With the advent of NGS in the form of WES and WGS, unprecedented amounts of genetic data are being generated, and primarily WGS represents an often-untapped data source, as an individual's complete PGx profile may be assessed while generating minimal additional costs. Due to decreasing sequencing costs, WGS is not prohibitively expensive for routine application and 60× WGS represents the superior solution (compared to 30× WGS and WES, Supplementary Figure S2) not only for diagnostics but also for PGx testing [14,23]. We substantiate this notion by showing that several known intronic or 
promoter DPWG variants (CYP2C19*17, CYP3A5*3, UGT1A1*28*37,VKORC1*2) are not covered in gnomAD exomes due to insufficient WES capturing (depending on the used WES capturing kit), and thus incomplete treatment recommendations may be provided based on WES. Using one of several existing NGS panels, including PGRNseq [7], AmpliSeq Pharmacogenomics Research Panel (thermofisher.com), or VeraCode ADME Core Panel (illumina.com), these variants indeed would be interrogated, but at the cost of requiring an additional test. Considering that every individual on average harbors six pharmacogenetically actionable variants, we identified several patients, who require significant PGx-based dosage adjustments, which has been shown to optimize treatment outcome for instance for the anticoagulants phenprocoumon or warfarin [24].

Second, the recent technological advances have caused a paradigm shift and, currently, interpretation of (pharmaco)genetic data represents the greater challenge than their generation. In order to streamline analysis and facilitate interpretation, most providers resort to genotyping of a number of variants, of which effects on phenotype are established and translated into clear clinical guidelines. The caveat of this approach is that only the small subset of variants in the genes are interrogated, potentially missing yet undescribed or rare variants, resulting in incorrect drug recommendations. Indeed, a rare/novel LOF variant occurring in cis with an ultrarapid-metabolizer variant would lead to misclassification as ultrarapid metabolizer and hence lead to incorrect PGx recommendations. To estimate such cases, in gnomAD and our in-house WGS cohort we assessed the relative allele frequencies of DPWG and LOF/missense variants highly likely to disrupt the function of DPWG pharmacogenes. Thereby, we showed that the 45 analyzed DPWG variants, when combined, indeed comprise $\sim 98 \%$ of detected pharmacogene alleles, confirming and expanding the results of two recent studies analyzing the much smaller datasets of the 1000 Genomes Project, Exome Sequencing Project, and ExAC $[13,25]$. As expected, the vast majority of novel LOF/missense variants occurred with a MAF $<0.1 \%$, or even with an allele count of 1 . Thus, the screening of DPWG variants is of high clinical utility and cost efficient, may even be performed for individuals assessed in large-scale biobanks [19], but is ultimately limited in its scope. With increasing sequencing data available, future efforts are warranted to focus on sequencing-based approaches to enable better precision medicine, however, thereby generating novel challenges such as the interpretation of variants of unknown significance (VUS) in pharmacogenes. In the event of additional PGx variants being described, we may re-analyze our WGS data and subsequently integrate such variants into the patients' updated PGx profiles, which are accessible using the QR code on the MSC.

Third, short-read sequencing is inherently limited in repetitive and/or homologous genomic regions (i.e., mappability $<1$; the so-called "dead zone" of the genome) [14], hampering accurate variant calling, for example in CYP genes. The most prominent example is the CYP2D6 gene, responsible for bioactivation or elimination of $25 \%$ of prescribed drugs [26], and its adjacent pseudogenes CYP2D7 and CYP2D8P. Several of the 139 star alleles currently listed in PharmVar (pharmvar.org/gene/CYP2D6) are possible to detect, however, the number of duplications and gene rearrangements leading to CYP2D6-CYP2D7 hybrid genes are notoriously difficult to genotype using short-read-based NGS approaches. To resolve these complex genotypes from short-read NGS data, freely available software tools, such as used in this study and others [18], have been introduced. Notwithstanding the powerful algorithms behind the tested tools Astrolabe, Aldy, and Stargazer, none of them assigned the correct GeT-RM 2019 consensus genotypes (generated using a variety of orthogonal methods) to all of the tested samples. In general, the tools accurately called most of the samples with simple CYP2D6 genotypes and with only two star alleles. Discordantly called genotypes occurred in complex SVs, where Aldy reached the overall highest accuracy, not excluding the possibility that Aldy might have been trained using some of the GeT-RM samples and that the GeT-RM genotypes might be incomplete. Thus, primarily Aldy represents an important addition to the NGS software toolkit. Although incorporating bioinformatic CYP2D6 analysis from short-read NGS data is a timesaving alternative to wetlab approaches, further evaluation of such software tools (e.g., by using long-read sequencing) is warranted. Another key challenge of PGx implementation using microarrays or short-read sequencing 
into actionable recommendations is phasing of variants. Several phasing algorithms exist, such as Beagle (implemented in Stargazer) [27], FastPHASE [28], and SHAPE-IT [29], all of which rely on statistical inference to assign diplotypes. The most efficient and straight forward method for variant phasing, however, is long-read sequencing, using e.g., PacBio (pacb.com) or Oxford Nanopore Technologies (nanoporetech.com). It has been shown that targeted long-read sequencing of the CYP2D6 gene enables phasing and accurate calling of SNVs, indels, and SVs [30-32], which could be further evaluated using long-read WGS or hybrid assemblies of short and long reads.

Fourth, providing the PGx information as the MSC [10] or the recently developed "PGx-Passport" [33] in addition to a written report, if used correctly, allows platform-independent implementation of pharmacogenetics and patient autonomy. In theory, by integrating information on individual PGx profiles for decision support of drug selection and dosage, ADE, costs, and time can be reduced, the extent of which is currently being investigated in the PREPARE trial [9]. The Netherlands takes on a pioneering role in the implementation of pharmacogenetics, integrating PGx information in the Dutch G-Standard database, which is used by all parties in the healthcare system and provides the PGx recommendation text if a drug is prescribed or purchased [34].

Fifth, not only healthcare systems, but also the drug development industry may benefit from the implementation of PGx profiling. Currently, only $10 \%$ of all drugs that initiate phase I clinical trials are subsequently approved [2], among other reasons potentially due to neglected patient stratification such as according to metabolizer status in clinical trials. This caveat is exemplified by the angiotensin-II-type-1-receptor-antagonist losartan, which showed highly promising results in preclinical studies [35], which, however, could not be replicated in large-scale clinical trials for Marfan syndrome [36]. Because $\sim 14 \%$ of losartan is oxidized by CYP2C9 into its $10-40 \times$ more potent metabolite E-3174, it is assumed that the majority of the effect of losartan stems from E-3174 [37]. For individuals with the frequently occurring slow metabolizer alleles CYP2C $* 2, * 3, * 5$, therefore an increased dosage of losartan might be necessary to achieve a therapeutic effect, which ultimately might explain the discrepancies between preclinical and (non-pharmacogenetically-stratified) clinical trials with losartan. Indeed, clinical trials might benefit from increased stratification according to PGx status, similarly as stratification is more common in oncology trials according to tumor driver variants [6].

We acknowledge following limitations of our study: The main limitation of our study is that for gnomAD, individual-level information is (yet) unavailable and thus we could not screen for co-occurring PGx variants in the same individual. In addition, because the here analyzed sequencing data were generated using short-read NGS, information on variant phasing is unavailable and therefore, to infer wildtype $\left({ }^{*} 1\right)$ alleles, we assumed that variants detected in the same gene occur in trans, likely leading to an underestimation of wildtype alleles. Finally, as we limited our analysis to DPWG variants and LOF/missense variants, we likely missed other, pharmacogenetically-relevant variants, which, however, are difficult to interpret with the current knowledge. Considering these limitations, our calculations should be regarded as estimates, aiming to show the frequency of variant alleles in large-scale datasets.

\section{Materials and Methods}

\subsection{PGx Profiling from WGS Data as well as Comparison of WGS and WES for PGx Profiling}

WGS (PCR-free, 60× 150PE) of 547 individuals with rare, mainly cardiovascular or connective tissue disorders was performed as previously described [23]. Resulting raw data in the form of FASTQ files were aligned, generating binary alignment map (BAM) files, and variant calling of SNVs and small indels, generating variant call format (VCF) files, was performed using GENALICE MAP v2.5.6 (Genalice, Nijkerk, The Netherlands) as previously described [38]. For 11 DPWG pharmacogenes (CYP2B6, CYP2C9, CYP2C19, CYP2D6, CYP3A5, DPYD, F5, SLCO1B1, TPMT, UGT1A1, VKORC1) and an $H L A-B^{*} 5701$ tagging variant, common/known variants (i.e., known to generate DPWG guidelines) as well as rare/novel but potentially relevant LOF variants (SNVs and indels) were extracted from 
our 547 in-house genomes using GENALICE MAP's gaVariant module, generating small gVCF-like VCF files displaying homozygous reference alleles as well (Supplementary Table S2). Note that other variant calling tools such as GATK [39] may also be used to extract pharmacogenetically-relevant variants and to generate small gVCF-like VCF files. To generate a personalized PGx report and MSC according to the DPWG guidelines, SNVs, indels, and possible SVs (such as affecting CYP2D6) were combined into a single VCF file and used as input for the Genetic Information Management System (GIMS) portal of bio.logis (bio.logis Genomic Healthcare GmbH, FFM, Germany).

Moreover, for the 45 DPWG variants, all the coding exons in the 11 current DPWG genes, and the core and extended ADME genes listed in pharmaadme.org, we compared the read-depth coverage of $60 \times$ and $30 \times$ WGS (TruSeq DNA PCR-Free; Illumina Inc., San Diego, CA, USA) as well as 100× WES (SureSelect Human all Exon v6 and v7; Agilent Technologies Inc., Santa Clara, CA, USA) considering the PGx profiling of 5 samples each (see Supplementary Methods and Supplementary Figure S2) [40].

\subsection{Analysis of Star Alleles and Loss-of-Function Variants in gnomAD and in Our In-House WGS Cohort}

The corresponding VCF files of gnomAD exomes v2.1.1 (125'748 exomes, released 2017, genome build GRCh37/hg19) and gnomAD genomes v3 (71'702 genomes, released 2019, genome build GRCh38/hg38) were downloaded (gnomAD.broadinstitute.com/downloads; note that as the genomes of gnomAD v2.1.1 are largely incorporated in the genomes of gnomAD v3, we considered only gnomAD v3 for genomes). In our cohort containing 547 WGS samples, joint variant calling was performed using the Population Calling module of GENALICE MAP [38] to simultaneously extract all sequence variants in 11 DPWG pharmacogenes and an $H L A-B^{*} 5701$ tagging variant, generating a multi-sample VCF file. Note that due to short-read-related alignment ambiguities, LOF and missense variants in HLA-B were not considered and the HCP5 variant rs2395029 (Chr6(GRCh37):g.31431780T>G) was used as marker linked to the $H L A-B^{*} 5701$ allele in populations with European ancestry [41].

The conversion of gnomAD v3 genomic positions from GRCh38/hg38 to GRCh37/hg19 (i.e., liftover) as well as the subsequent annotation and filtering of VCF files derived from gnomAD (v2.1.1 and v3) and our in-house WGS cohort was performed using VarSeq v2.2.0 (Golden Helix Inc., Bozeman, MT, USA). To restrict the analysis to high-confidence variant calls, we excluded sequence variants with a non-PASS gnomAD filter and, as 150-bp-long reads were used, such with 150-mer mappability $<1$ (calculated using GEM version GEM-binaries-Linux-x86_64-20100419-003425, with $m=2$ ) [14,42].

To determine and compare relative allele frequencies of pharmacogenetically-relevant variants in gnomAD exomes v2.1.1, genomes v3, and our in-house cohort, we applied multiple filtering criteria. Using VarSeq's automated filter functions, we filtered for the 45 SNVs and indels implemented in the PREPARE trial [9] (i.e., the most well described/known PGx variants with DPWG guidelines) as well as for variants potentially affecting gene function: (i) Loss-of-function (LOF) variants, defined as premature termination codons (PTCs) caused by nonsense and frameshift mutations with or without expected nonsense-mediated mRNA decay, as well as canonical splice site variants (intronic $\pm 1-2 \mathrm{bp}$ ) caused by single nucleotide changes and in silico predicted to alter splicing; (ii) missense variants classified as "damaging" or "deleterious" by all six corresponding in silico prediction tools (FATHMM, FATHMM-MKL, MutationAssessor, MutationTaster, Polyphen2, SIFT) as previously described [43]. The resulting lists of LOF and missense variants were subsequently filtered for variants listed in ClinVar (v2019.11; ncbi.nlm.nih.gov/clinvar) as "Drug Response" [44] and/or in the Human Gene Mutation Database (HGMD) professional (v2019.4; portal.biobase-international.com) as "FP", "DFP", "DM?", or "DM" in the context of drug response [45], and/or the PharmGKB database (v2019.6; pharmgkb.org/downloads) [46].

For all 11 analyzed pharmacogenes, relative allele frequencies of DPWG and LOF variants were assessed and compared among gnomAD v2.1.1, v3, and our in-house WGS cohort. To infer the number of wildtype $\left({ }^{*} 1\right)$ alleles, we subtracted the number of detected DPWG and LOF alleles from the total allele numbers in gnomAD v3, v2.1.1, and our in-house cohort under the assumption that variants detected in the same gene occur in trans. In addition, in our in-house WGS cohort, but not in gnomAD, 
we were able to assess the co-occurrences of DPWG, LOF and/or likely pathogenic missense variants in the same individual.

\subsection{Evaluation of CYP2D6 Variant Callers}

For the SV detection in CYP2D6, we evaluated the software tools Astrolabe (previously Constellation) v0.8.6.1 [15], Aldy v2.2.3 [16], and Stargazer v1.0.7 [17]. To compare the accuracy of these three variant callers, we downloaded FASTQ and/or BAM files of 20 human PGx reference WGS samples from the European Nucleotide Archive (ENA, ebi.ac.uk) (HG00436, NA07029, NA18959, NA19109, NA21781, NA12873, NA18861, HG00589, NA19917, NA07019, NA12717, HG00276, NA18524, NA18540, NA07348, NA18519; NA18966, NA18992 and NA19226) [47] or from ftp-trace.ncbi.nih.gov/1000genomes/ftp/phase3/data/NA12892 (NA12892). Variant calling was performed using GENALICE MAP [38] for all but one (NA12892) of these downloaded samples. For NA12892, we used Strelka2 v2.9.10 [48] because only the BAM file was available for download (cf. GENALICE MAP requires FASTQ file as input). In addition, for the reference sample NA12878 (termed NA12878 in-house), we analyzed the genomic DNA by means of our in-house WGS pipeline (PCR-free, $60 \times$ PE150) using Isaac v01.15.02.08 for alignment and v2.0.13 for variant calling [49]. For the software tool Aldy, only BAM files were required as inputs, while the tool Astrolabe needed VCF and BAM files. For Stargazer, in addition to BAM files, GATK-DepthOfCoverage format (GDF) files were required as input (generated using GATK v3.5) and RYR1 served as control gene. All three SV detection tools were applied using default settings (Supplementary Figure S4). For each reference sample, the results of SV calling were compared to the consensus CYP2D6 genotype obtained from the GeT-RM projects 2019 [21]. For resolving ambiguous calls, BAM files were manually evaluated using the Integrative Genomics Viewer v2.4.19 [22]. Moreover, we used Aldy [16] to assess the frequencies of CYP2D6 star alleles in our in-house WGS cohort.

\subsection{Statistical Analysis}

The upper and lower limits of $95 \%$ confidence intervals of relative frequencies were calculated using the online tool VassarStats with a correction for continuity (vassarstats.net/prop1.html).

\section{Conclusions}

Taken together, we show that short-read WGS, rather than WES, is suitable for the profiling of pharmacogenes, including CYP2D6. WGS-based clinical sequencing may therefore be the most comprehensive all-in-one approach for the simultaneous testing of Mendelian diseases and profiling of pharmacogenes without generating significant additional costs. Moreover, we demonstrate that known DPWG variants comprise the majority of PGx variation. Hence, restricting PGx profiling to these variants streamlines the interpretation process and provides appropriate pharmacogenetically-guided treatment recommendations for the vast majority of individuals. For true precision medicine, i.e., for the best possible pharmacogenetically-guided treatment recommendations for each patient, however, it is warranted that comprehensive approaches, such as presented here, expand the targeted profiling of known DPWG variants to the genome-wide profiling of all pharmacogenes.

Supplementary Materials: Supplementary materials can be found at http://www.mdpi.com/1422-0067/21/7/2308/ s1.

Author Contributions: Conceptualization, S.M.C., J.M., and G.M.; data curation, S.M.C., T.S., and J.M.; formal analysis, G.M.; funding acquisition, G.M.; investigation, S.M.C., T.S.; methodology, S.M.C., T.S., J.M., and G.M.; project administration, G.M.; resources, G.M.; software, S.M.C. and T.S.; supervision, G.M.; validation, S.M.C., T.S., J.M., and G.M.; visualization, S.M.C., T.S.; writing—original draft preparation, S.M.C., T.S.; writing-review and editing, S.M.C., T.S., J.M., and G.M.; All authors have read and agreed to the published version of the manuscript.

Funding: This research was funded by the Clariant Foundation, Fondation pour la Recherche et le Traitement Médical (FRTM), Ernst Göhner Stiftung, Gebauer Stiftung, and Lotteriefonds ZH. 
Acknowledgments: We thank Stephan Fees from bio.logis (bio.logis Genomic Healthcare GmbH, FFM, Germany) for discussions and assistance with the Genetic Information Management System (GIMS) as well as Nicolo Dubacher for his valuable inputs and proofreading of the manuscript.

Conflicts of Interest: The authors declare no conflict of interest. The funders had no role in the design of the study; in the collection, analyses, or interpretation of data; in the writing of the manuscript, or in the decision to publish the results.

\section{References}

1. Relling, M.V.; Klein, T.E. CPIC: Clinical Pharmacogenetics Implementation Consortium of the Pharmacogenomics Research Network. Clin. Pharmacol. Ther. 2011, 89, 464-467. [CrossRef]

2. Dowden, H.; Munro, J. Trends in clinical success rates and therapeutic focus. Nat. Rev. Drug Discov. 2019, 18, 495-496. [CrossRef]

3. DiMasi, J.A.; Grabowski, H.G.; Hansen, R.W. Innovation in the pharmaceutical industry: New estimates of R\&D costs. J. Health Econ. 2016, 47, 20-33. [CrossRef]

4. Strengthening Pharmacovigilance to Reduce Adverse Effects of Medicines. Available online: Europa.eu/ rapid/press-release_MEMO-08-782_de.htm?locale=en (accessed on 24 January 2020).

5. Watanabe, J.H.; McInnis, T.; Hirsch, J.D. Cost of Prescription Drug-Related Morbidity and Mortality. Ann. Pharmacother. 2018, 52, 829-837. [CrossRef]

6. Filipski, K.K.; Mechanic, L.E.; Long, R.; Freedman, A.N. Pharmacogenomics in oncology care. Front. Genet. 2014, 5, 73. [CrossRef] [PubMed]

7. Gordon, A.S.; Fulton, R.S.; Qin, X.; Mardis, E.R.; Nickerson, D.A.; Scherer, S. PGRNseq: A targeted capture sequencing panel for pharmacogenetic research and implementation. Pharmacogenet. Genom. 2016, 26, 161-168. [CrossRef] [PubMed]

8. Swen, J.J.; Nijenhuis, M.; de Boer, A.; Grandia, L.; Maitland-van der Zee, A.H.; Mulder, H.; Rongen, G.A.P.J.M.; van Schaik, R.H.N.; Schalekamp, T.; Touw, D.J.; et al. Pharmacogenetics: From bench to byte-an update of guidelines. Clin. Pharmacol. Ther. 2011, 89, 662-673. [CrossRef] [PubMed]

9. van der Wouden, C.H.; Cambon-Thomsen, A.; Cecchin, E.; Cheung, K.C.; Dávila-Fajardo, C.L.; Deneer, V.H.; Dolžan, V.; Ingelman-Sundberg, M.; Jönsson, S.; Karlsson, M.O.; et al. Implementing Pharmacogenomics in Europe: Design and Implementation Strategy of the Ubiquitous Pharmacogenomics Consortium. Clin. Pharmacol. Ther. 2017, 101, 341-358. [CrossRef]

10. Blagec, K.; Koopmann, R.; Crommentuijn-van Rhenen, M.; Holsappel, I.; van der Wouden, C.H.; Konta, L.; $\mathrm{Xu}$, H.; Steinberger, D.; Just, E.; Swen, J.J.; et al. Implementing pharmacogenomics decision support across seven European countries: The Ubiquitous Pharmacogenomics (U-PGx) project. J. Am. Med. Inform. Assoc. 2018, 25, 893-898. [CrossRef] [PubMed]

11. Gottesman, O.; Scott, S.A.; Ellis, S.B.; Overby, C.L.; Ludtke, A.; Hulot, J.-S.; Hall, J.; Chatani, K.; Myers, K.; Kannry, J.L.; et al. The CLIPMERGE PGx Program: Clinical implementation of personalized medicine through electronic health records and genomics-pharmacogenomics. Clin. Pharmacol. Ther. 2013, 94, $214-217$. [CrossRef]

12. Rasmussen-Torvik, L.J.; Stallings, S.C.; Gordon, A.S.; Almoguera, B.; Basford, M.A.; Bielinski, S.J.; Brautbar, A.; Brilliant, M.H.; Carrell, D.S.; Connolly, J.J.; et al. Design and anticipated outcomes of the eMERGE-PGx project: A multicenter pilot for preemptive pharmacogenomics in electronic health record systems. Clin. Pharmacol. Ther. 2014, 96, 482-489. [CrossRef] [PubMed]

13. Kozyra, M.; Ingelman-Sundberg, M.; Lauschke, V.M. Rare genetic variants in cellular transporters, metabolic enzymes, and nuclear receptors can be important determinants of interindividual differences in drug response. Genet. Med. 2017, 19, 20-29. [CrossRef] [PubMed]

14. Caspar, S.M.; Dubacher, N.; Kopps, A.M.; Meienberg, J.; Henggeler, C.; Matyas, G. Clinical sequencing: From raw data to diagnosis with lifetime value. Clin. Genet. 2018, 93, 508-519. [CrossRef] [PubMed]

15. Twist, G.P.; Gaedigk, A.; Miller, N.A.; Farrow, E.G.; Willig, L.K.; Dinwiddie, D.L.; Petrikin, J.E.; Soden, S.E.; Herd, S.; Gibson, M.; et al. Constellation: A tool for rapid, automated phenotype assignment of a highly polymorphic pharmacogene, CYP2D6, from whole-genome sequences. NPJ Genom. Med. 2016, 1, 15007. [CrossRef] [PubMed] 
16. Numanagić, I.; Malikić, S.; Ford, M.; Qin, X.; Toji, L.; Radovich, M.; Skaar, T.C.; Pratt, V.M.; Berger, B.; Scherer, S.; et al. Allelic decomposition and exact genotyping of highly polymorphic and structurally variant genes. Nat. Commun. 2018, 9, 828. [CrossRef]

17. Lee, S.B.; Wheeler, M.M.; Thummel, K.E.; Nickerson, D.A. Calling Star Alleles With Stargazer in 28 Pharmacogenes With Whole Genome Sequences. Clin. Pharmacol. Ther. 2019, 106, 1328-1337. [CrossRef]

18. Tremmel, R.; Klein, K.; Battke, F.; Fehr, S.; Winter, S.; Scheurenbrand, T.; Schaeffeler, E.; Biskup, S.; Schwab, M.; Zanger, U.M. Copy number variation profiling in pharmacogenes using panel-based exome resequencing and correlation to human liver expression. Hum. Genet. 2020, 139, 137-149. [CrossRef]

19. Reisberg, S.; Krebs, K.; Lepamets, M.; Kals, M.; Mägi, R.; Metsalu, K.; Lauschke, V.M.; Vilo, J.; Milani, L. Translating genotype data of 44,000 biobank participants into clinical pharmacogenetic recommendations: Challenges and solutions. Genet. Med. 2019, 21, 1345-1354. [CrossRef]

20. VKORC1-Genotypisierung. Available online: Charite.de/fileadmin/user_upload/microsites/m_cc05/ilp/ referenzdb/20204037.htm (accessed on 24 January 2020).

21. Gaedigk, A.; Turner, A.; Everts, R.E.; Scott, S.A.; Aggarwal, P.; Broeckel, U.; McMillin, G.A.; Melis, R.; Boone, E.C.; Pratt, V.M.; et al. Characterization of Reference Materials for Genetic Testing of CYP2D6 Alleles: A GeT-RM Collaborative Project. J. Mol. Diagn. 2019, 21, 1034-1052. [CrossRef]

22. Robinson, J.T.; Thorvaldsdóttir, H.; Winckler, W.; Guttman, M.; Lander, E.S.; Getz, G.; Mesirov, J.P. Integrative genomics viewer. Nat. Biotechnol. 2011, 29, 24-26. [CrossRef]

23. Meienberg, J.; Bruggmann, R.; Oexle, K.; Matyas, G. Clinical sequencing: Is WGS the better WES? Hum. Genet. 2016, 135, 359-362. [CrossRef] [PubMed]

24. Gage, B.F.; Bass, A.R.; Lin, H.; Woller, S.C.; Stevens, S.M.; Al-Hammadi, N.; Li, J.; Rodríguez, T.; Miller, J.P.; McMillin, G.A.; et al. Effect of Genotype-Guided Warfarin Dosing on Clinical Events and Anticoagulation Control Among Patients Undergoing Hip or Knee Arthroplasty: The GIFT Randomized Clinical Trial. JAMA 2017, 318, 1115. [CrossRef] [PubMed]

25. Ingelman-Sundberg, M.; Mkrtchian, S.; Zhou, Y.; Lauschke, V.M. Integrating rare genetic variants into pharmacogenetic drug response predictions. Hum. Genom. 2018, 12, 26. [CrossRef] [PubMed]

26. Zhou, S.F. Polymorphism of human cytochrome P450 2 D6 and its clinical significance: Part I. Clin. Pharmacokinet. 2009, 48, 689-723. [CrossRef]

27. Browning, S.R.; Browning, B.L. Rapid and accurate haplotype phasing and missing-data inference for whole-genome association studies by use of localized haplotype clustering. Am. J. Hum. Genet. 2007, 81, 1084-1097. [CrossRef]

28. Scheet, P.; Stephens, M. A fast and flexible statistical model for large-scale population genotype data: Applications to inferring missing genotypes and haplotypic phase. Am. J. Hum. Genet. 2006, 78, 629-644. [CrossRef]

29. Delaneau, O.; Coulonges, C.; Zagury, J.-F. Shape-IT: New rapid and accurate algorithm for haplotype inference. BMC Bioinformatics 2008, 9, 540. [CrossRef]

30. Qiao, W.; Yang, Y.; Sebra, R.; Mendiratta, G.; Gaedigk, A.; Desnick, R.J.; Scott, S.A. Long-Read Single Molecule Real-Time Full Gene Sequencing of Cytochrome P450-2D6. Hum. Mutat. 2016, 37, 315-323. [CrossRef]

31. Buermans, H.P.J.; Vossen, R.H.A.M.; Anvar, S.Y.; Allard, W.G.; Guchelaar, H.-J.; White, S.J.; den Dunnen, J.T.; Swen, J.J.; van der Straaten, T. Flexible and Scalable Full-Length CYP2D6 Long Amplicon PacBio Sequencing. Hum. Mutat. 2017, 38, 310-316. [CrossRef]

32. Liau, Y.; Maggo, S.; Miller, A.L.; Pearson, J.F.; Kennedy, M.A.; Cree, S.L. Nanopore sequencing of the pharmacogene CYP2D6 allows simultaneous haplotyping and detection of duplications. Pharmacogenomics 2019, 20, 1033-1047. [CrossRef]

33. Van der Wouden, C.H.; van Rhenen, M.H.; Jama, W.O.M.; Ingelman-Sundberg, M.; Lauschke, V.M.; Konta, L.; Schwab, M.; Swen, J.J.; Guchelaar, H.-J. Development of the PGx-Passport: A Panel of Actionable Germline Genetic Variants for Pre-Emptive Pharmacogenetic Testing. Clin. Pharmacol. Ther. 2019, 106, 866-873. [CrossRef] [PubMed]

34. The G-Standaard: The Medicines Standard in Healthcare. Available online: Knmp.nl/producten/ gebruiksrecht-g-standaard/informatie-over-de-g-standaard/the-g-standaard-the-medicines-standard-inhealthcare (accessed on 10 January 2020). 
35. Habashi, J.P.; Judge, D.P.; Holm, T.M.; Cohn, R.D.; Loeys, B.L.; Cooper, T.K.; Myers, L.; Klein, E.C.; Liu, G.; Calvi, C.; et al. Losartan, an AT1 antagonist, prevents aortic aneurysm in a mouse model of Marfan syndrome. Science 2006, 312, 117-121. [CrossRef] [PubMed]

36. Lacro, R.V.; Dietz, H.C.; Sleeper, L.A.; Yetman, A.T.; Bradley, T.J.; Colan, S.D.; Pearson, G.D.; Selamet Tierney, E.S.; Levine, J.C.; Atz, A.M.; et al. Atenolol versus Losartan in Children and Young Adults with Marfan's Syndrome. N. Engl. J. Med. 2014, 371, 2061-2071. [CrossRef] [PubMed]

37. Sica, D.A.; Gehr, T.W.B.; Ghosh, S. Clinical pharmacokinetics of losartan. Clin. Pharmacokinet. 2005, 44, 797-814. [CrossRef]

38. Plüss, M.; Kopps, A.M.; Keller, I.; Meienberg, J.; Caspar, S.M.; Dubacher, N.; Bruggmann, R.; Vogel, M.; Matyas, G. Need for speed in accurate whole-genome data analysis: GENALICE MAP challenges BWA/GATK more than PEMapper/PECaller and Isaac. Proc. Natl. Acad. Sci. USA 2017, 114, E8320-E8322. [CrossRef]

39. Van der Auwera, G.A.; Carneiro, M.O.; Hartl, C.; Poplin, R.; Del Angel, G.; Levy-Moonshine, A.; Jordan, T.; Shakir, K.; Roazen, D.; Thibault, J.; et al. From FastQ data to high confidence variant calls: The Genome Analysis Toolkit best practices pipeline. Curr. Protoc. Bioinform. 2013, 43, 1-33. [CrossRef]

40. Meienberg, M.; Zerjavic, K.; Keller, I.; Okoniewski, M.; Patrignani, A.; Ludin, K.; Xu, Z.; Steinmann, B.; Röthlisberger, B.; Schlapbach, R.; et al. New insights into the performance of human whole-exome capture platforms. Nucleic Acids Res. 2015, 43, e45. [CrossRef]

41. Colombo, S.; Rauch, A.; Rotger, M.; Fellay, J.; Martinez, R.; Fux, C.; Thurnheer, C.; Günthard, H.F.; Goldstein, D.B.; Furrer, H.; et al. The HCP5 Single-Nucleotide Polymorphism: A Simple Screening Tool for Prediction of Hypersensitivity Reaction to Abacavir. J. Infect. Dis. 2008, 198, 864-867. [CrossRef]

42. Derrien, T.; Estellé, J.; Marco Sola, S.; Knowles, D.G.; Raineri, E.; Guigó, R.; Ribeca, P. Fast computation and applications of genome mappability. PLoS ONE 2012, 7, e30377. [CrossRef]

43. Najafi, A.; Caspar, S.M.; Meienberg, J.; Rohrbach, M.; Steinmann, B.; Matyas, G. Variant filtering, digenic variants, and other challenges in clinical sequencing: A lesson from fibrillinopathies. Clin. Genet. 2020, 97, 235-245. [CrossRef]

44. Landrum, M.J.; Lee, J.M.; Riley, G.R.; Jang, W.; Rubinstein, W.S.; Church, D.M.; Maglott, D.R. ClinVar: Public archive of relationships among sequence variation and human phenotype. Nucleic Acids Res. 2014, 42, D980-D985. [CrossRef] [PubMed]

45. Stenson, P.D.; Mort, M.; Ball, E.V.; Shaw, K.; Phillips, A.; Cooper, D.N. The Human Gene Mutation Database: Building a comprehensive mutation repository for clinical and molecular genetics, diagnostic testing and personalized genomic medicine. Hum. Genet. 2014, 133, 1-9. [CrossRef] [PubMed]

46. Thorn, C.F.; Klein, T.E.; Altman, R.B. PharmGKB: The Pharmacogenomics Knowledge Base. Methods Mol. Biol. 2013, 1015, 311-320. [CrossRef] [PubMed]

47. Pratt, V.M.; Everts, R.E.; Aggarwal, P.; Beyer, B.N.; Broeckel, U.; Epstein-Baak, R.; Hujsak, P.; Kornreich, R.; Liao, J.; Lorier, R.; et al. Characterization of 137 Genomic DNA Reference Materials for 28 Pharmacogenetic Genes: A GeT-RM Collaborative Project. J. Mol. Diagn. 2016, 18, 109-123. [CrossRef]

48. Kim, S.; Scheffler, K.; Halpern, A.L.; Bekritsky, M.A.; Noh, E.; Källberg, M.; Chen, X.; Kim, Y.; Beyter, D.; Krusche, P.; et al. Strelka2: Fast and accurate calling of germline and somatic variants. Nat. Methods 2018, 15, 591-594. [CrossRef]

49. Raczy, C.; Petrovski, R.; Saunders, C.T.; Chorny, I.; Kruglyak, S.; Margulies, E.H.; Chuang, H.-Y.; Källberg, M.; Kumar, S.A.; Liao, A.; et al. Isaac: Ultra-fast whole-genome secondary analysis on Illumina sequencing platforms. Bioinformatics 2013, 29, 2041-2043. [CrossRef]

(C) 2020 by the authors. Licensee MDPI, Basel, Switzerland. This article is an open access article distributed under the terms and conditions of the Creative Commons Attribution (CC BY) license (http://creativecommons.org/licenses/by/4.0/). 